\title{
Well-Being Measurement Scale For Farmers In Northeast Thailand
}

Banjong Krobbuaban, M.D., Ph.D., Chaiyaphum Hospital, Thailand Buapun Phompakping, Ph.D., Khon Kaen University, Thailand

\begin{abstract}
The purpose of this research study was to develop a well-being scale for the farmers of the northeastern region of Thailand. This descriptive research employed a workshop for ten qualified representatives of the personnel-selected by purposive sampling-from both the government and private sectors working with farmers in Chaiyaphum Province. The workshop was designed to find solutions for meanings, factors, indicators, and a scale of well-being of the farmers in the Northeast based on data from previous research on the meanings and well-being indicators of northeastern farmers. Our Scale was drafted and then examined in terms of its content validity, construction validity, reliability and the norm of the scale. Twenty-one experts evaluated the content validity. Data were collected from 1,600 farmers living in Chaiyaphum Province by multi-stage random sampling. Factor analysis and Cronbach's alpha coefficient were performed. The Northeastern Farmers' Wellbeing Scale (NFW Scale) was successfully completed and included 79 items in 12 domains with 49 indicators; including: illness (2), physical and health services (6), mental state (3), mental power (3), social (4), spiritual and beliefs (7), knowledge (4), family (5), economics (4), financial (4), housing (4) and environmental management (3). The reliability was 0.93. The overall scores of the NFW Scale were assigned to 3 groups using a normative model: 'better well-being' $\geq 299$; average between 260 and 298 ; and, 'below average' $\leq 259$.
\end{abstract}

Keywords: Farmers' Wellbeing Scale; Thai Farmers; Chaiyaphum Province

\section{INTRODUCTION}

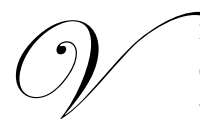

irtually no nation has been exempted from the pressures and strictures of the unrelenting economic competition that characterizes globalization. Governments are obliged to adopt developmental policies; with a particular emphasis on the economic dimension, which in turn affects every other aspect of society. Notwithstanding the importance of economic development, there are other factors that contribute to people's wellbeing; an overemphasis on economic indicators can lead to psychosocial damage, like abnormal behaviours and the loss of equanimity. Such phenomena do not reflect the emergence of a better quality of life or greater happiness; as people's wellbeing is not dependent on the country's economic condition; for as Layard (2005) points out although the per capita income in western countries in the past 50 years has doubled but the level of satisfaction of life has not increased. Nevertheless, in developing countries, beyond food security, it is people's economic "lack" which is a persistent, major barrier to happiness. We therefore need to discover the meaning of development particularly with respect to a model in which people "choose" what they desire to give them a sense of wellbeing.

Thailand's economic development in the past emphasized economic expansion and stability. This approach successfully tackled the income facet of the poverty problem and most people had better means of making a living. The lack of wealth has decreased, but the problem of inequality of income distribution remains: the gap between the rich and the poor has widened because the economic expansion benefitted the rich more than the disadvantaged. The gap between the modern urban economy and the rural agricultural sector can also be clearly seen. 
Thus it is the farmers who have been suffering the most but exacerbated by the lack of economic and social support for rural areas based on a developmental philosophy that is focused on exploiting resources and energy, for trade in the global market place. Thailand is faced with two challenges: one is to be self-sufficient and the other is to be competitive in a world dominated by mega economies (viz., the USA, the EU and China). The mercurial world economic situation has had a measureable negative impact on the wellbeing of Thai people - unhealthy working conditions/expectations and environmental degradation/pollution.

Satisfaction with respect to development has received both domestic and international attention (Sen, 1999). Thus, academics and policymakers have coined a term for the human response to development as 'degree of happiness', 'quality of life', and 'wellbeing'. Though the concept of wellbeing has been developed to some extent, further clarification is needed. Wellbeing rating scales have been developed and can be used to help frame issues needing redress in different terms and help to reveal the various components that can be isolated and ameliorated. Importantly, the concept acknowledges the importance of the local context and local participation (Krobbuaban, 2011). At present, however, there is no tool or wellbeing measuring scale for use among the farmers and rural communities of northeastern Thailand.

\section{THE OBJECTIVE}

The objective is to develop a rating scale to measure wellbeing among the rural, agrarian population of the northeastern geopolitical region of Thailand.

\section{METHODS}

A wellbeing measurement scale for farmers in northeastern Thailand was begun by gathering a list of questions covering the range of wellbeing indicators, agreed upon during group discussions at a workshop of qualified individuals (Krobbuaban, 2011). The researcher revised the questions so that farmers in the sample group would understand. Then a Likert rating scale, consisting of five levels, was developed: for positive questions 1, 2, 3, 4 and 5 indicated 'least', 'little', 'moderate', 'much', and 'most', respectively. For negative questions, the rating was reversed so that 5, 4, 3, 2 and 1 indicated 'least', 'little', 'moderate', 'much' and 'most', respectively.

Twenty-one experts from relevant disciplines, religious leaders and village wise men then assessed the wellbeing measurement scale for content validity. The data were analyzed for the median and interquartile range (IQR). The accepted median was $\geq 3.5$ and the IQR ranged between Quartile T1 and Quartile T3, which was $<1.50$ (Gosa, 1999). The feedback suggestions were synthesized and the wellbeing measurement scale revised before returning it to the validation experts for reassessment until every question was unanimously approved.

Then the researcher conducted pilot testing with 50 farmers whose characteristics were similar to those of the sample group to test the appropriateness of the language and the format of the questions before using it to collect data. Whenever a deficiency was found, it was corrected and revised. The revised scale was tested for validity according to construct validity theory. The sample group was calculated from the ratio of one question per at least 10-15 people. Thus, the sample group included between 790 and 1,185 people.

For this study, the researcher collected data from 1,600 farmers in Chaiyaphum by means of multi-stage cluster sampling. Chaiyaphum Province comprises 16 district; one hundred samples were selected from each sampling village. According to Comrey and Lee's classification, our sample size was appropriate (Comrey and Lee, 1992). After collecting the data, the researcher and the assistant keyed them into the computer in duplicate. The data were rechecked for correctness then analyzed for construct validity and factor analysis. The score range of values for wellbeing for northeastern farmers was calculated using the median and percentiles T25 and T75.The validity of the wellbeing scale was analyzed by using a computer package.

\section{RESULTS}

In all, there were 63 questions in our scale. Based on the research results related to each question, the researcher revised the language and assigned a positive or negative Likert scale. Twenty-one experts assessed the 
content validity. Seventeen experts $(80.95 \%)$ returned the result of their examination and suggestions. The researcher used these to calculate the median and the quartile range and interpreted the results. The median was $\geq$ 3.5 and the IQR was $<1.5$, which meant that the experts unanimously approved the appropriateness for a wellbeing measurement scale of farmers in Northeast Thailand.

Of 63 questions, 49 met the specified criteria while 14 did not. The invalid questions were revised by (a) deleting some questions (b) revising the language and/or (c) adding some questions to some factors. The final measurement scale comprised 79 questions; which were sent to the validation review experts for approval.

The researcher then tested the wellbeing measurement scale in a pilot of 50 farmers, who were not part of the research sample and checked the scale for language appropriateness and practicability. Farmers answered the questions by themselves and it was found that most could answer as per the instructions. The use of a mark for some choices was problematic for some respondents. When the researcher asked for a reason, they said that the space between the choices was too narrow. Thus, they did not realize that they had double marked the same question and had not marked the next one. Hence, the researcher widened the space for easier marking.

Most farmers in the sample group were females (61.0\%), between 36 and 45 years of age (36.5\%), married (84.2\%), housewives $(48.2 \%)$, and had elementary education $(56.1 \%)$. The largest minority income range was 20,000-29,999 baht per year (21.7\%) from agricultural work vs. others $(85.1 \%)$. Some were in debt (between 20,000 and 29,999 baht per year (17.4\%); ostensibly for agricultural and nonagricultural investment/expenses $(47.0 \%)$.

The findings of the survey on the structure of wellbeing factors among Northeast farmers - by means of exploratory factor analysis - revealed that their wellbeing included 12 factors. The first was two factors relating to illness: (1) being without chronic illness or disability and (2) being without a disease. The second was related to physical and health services with six indicators: (1) being able to go to work regularly, (2) health promotion and prevention, (3) health security (medicare rights), (4) fast services, (5) efficiency of services, and (6) home visits after hospitalization. The third was mental state with three indicators: (1) sufficiency, being satisfied with what they have and what they are, (2) being proud of themselves, and (3) being satisfied with their work. The fourth was mental capacity with three indicators: (1) mental readiness, (2) stress management, and safe surroundings. The fifth was social wellbeing with four indicators: (1) participation, (2) generosity, benevolence and sympathy, (3) unity and (4) community's emergency services. The sixth was spiritual cum religion including seven indicators (1) belief in Buddhist ways, (2) belief in holiness and the supernatural, (3) having religious precepts, (4) making merit, (5) having moral principles, (6) helping others and society, and (7) having wisdom. Knowledge wellbeing was the seventh factor comprising four indicators: (1) health building, (2) disease prevention, (3) knowledge on the disease one has and how to take care of oneself, and (4) knowledge on how to appropriately encounter various situations. The eighth factor was family with five indicators including (1) warmth in the family, (2) all of the family members living together, (3) family members taking care of each other in infirmity or old age, (4) happiness, and (5) having heirs. The ninth factor was economics with four indicators: (1) having equipment/instruments for earning a living, (2) having water resources, (3) having career wisdom, and (4) absence of natural hazards. The tenth factor was financial with four indicators including (1) having enough income, (2) having savings, (3) not having any debt, and (4) sufficient income to meet expenses. The eleventh was housing with four indicators: (1) cleanliness at home, (2) absence of disease sources/vectors and a focus on disease prevention, (3) having hygienic toilets, and (4) concern for care of the environment. The twelfth factor was environmental management with three indicators including (1) trash/refuse management, (2) use of biofertilizers, and (3) having social outlets. The reliability of the study was 0.93.The researcher used a Descriptive Model as the Normative Model and statistical means for testing the results of this research with its non-normal distribution. The median, Percentile T25 and Percentile T75 were used to define the norm according to the following scores: $\geq 300=$ better than the norm; $260-299=$ average/normal; and, $\leq 259$ =lower than the norm.

\section{DISCUSSION}

In the current study, the researcher collected questions from a workshop of qualified representatives of the target population. The questions selected had to be related to each indicator of each factor of the wellbeing of 
northeastern farmers. For each indicator, one or more questions could be made to capture the respondent's thoughts on a matter. Our questions were uniquely and neatly related to indicators of wellbeing agreed to by northeastern farmers and in three-dimensions (viz., 'having', 'wanting to be' and 'wanting to do') (Krobbuaban, 2011). In addition, the questions were integrative of the concepts and theories of wellbeing research, which would enable comparisons with other similar research in Thailand and around the world.

Based on the questions obtained from qualified representatives, the researcher constructed a wellbeing measurement scale for farmers in Northeast Thailand. Since the questionnaire included several questions covering indicators from both a positive and negative perspective, each question was rated using a Likert-type scale (1 to 5). On variable scales there is no zero (Panpruek, 2003), so we did not provide' 0 ' as a rating even though some responses could be 'no' or 'not at all'; for example, for answering the question, "Do you face a problem of insomnia?"

After that, the researcher studied the content validity to see if the tool covered all the aspects needed to accurately measure wellbeing. Experts having expertise on content performed the analysis then their suggestions were used to do revisions. This process was repeated until the entirety was unanimously approved (Ganasoot, 1995; Prasitratasin, 1997; Kheawyoo, 2000).

Twenty-one experts were selected to assess content validity; 17 of whom (81\%) completed the task. Two tests of validation were used: (1) Construct validity: expert judgment determined whether all the issues had been covered. In case of disagreement, the particular item or question was revised. Once unanimity was achieved, the scale was considered valid. (2) Statistical validity: the median and interquartile range (IQR) were calculated and an interpretation of the acceptance for each question was made according to specified criteria (i.e., median $\geq 3.5$ and IQR <1.5) (Gosa, 1999).

The content validity examination revealed that 49 questions met the criteria while 14 did not. The latter had an IQR $\geq 1.5$ and did not receive unanimous assent from the experts. Notwithstanding, the researcher had chosen questions because they could be used to analyze various specific aspects of each question, such as: (a) calculating the index value of agreement between the question and behavioral characteristics (b)determining the suitability index between the question and characteristics of the behavioral group (c) frequency distribution of each question (d) analyzing the correlation among the questions (e) analyzing the correlation between the score of each question and the total score (f) analyzing the internal correspondence of each question and (g) factor analysis.

Thus, the researcher reexamined these questions by putting emphasis on the data the farmers raised during focus conversations and synthesizing the experts' suggestions before revising the language used in, or the content of, each question. The end result was that some questions were omitted and new ones added in order to achieve the greatest content validity. There after the questionnaire was sent for reexamination until it was unanimously approved, indicating that having content validity was a priority.

Finally, the northeastern wellbeing measurement scale comprised 79 questions and 49 indicators. Its construct validity was confirmed by 1,600 farmers in Chaiyaphum Province. Factor analysis was used to determine whether the measured phenomena were valid and how much - in terms of theoretical or construct factors - was accurate. The persistent challenge of the study was assigning factors that were consistently and accurately referents of wellbeing.

The researcher analyzed the surveyed factors and discovered that wellbeing among northeastern farmers comprised 12 factors, 49 indicators, assessed through 79 questions. In the end, none of the questions were omitted. The correlation between the variables ranged between 0.33 and 0.91 , which could be used for further analysis of factors. When tested in the field, the farmers' wellbeing was categorized into 12 factors according to the correlation of variables, and those which were closely correlated were put into the same category. The variables for different factors were not correlated or only nominally.

The current study revealed that 12 factors of the northeastern farmers' wellbeing were clear. The reliability of the Cronbach's alpha coefficient was 0.93 , which was high ( 0.80 or above) and acceptable (Luecha, 1989; 
Kheawyoo, 2000; Prasitratasin, 1997); perhaps because our wellbeing measurement scale had gone through several stages of testing

\section{CONCLUSION}

The wellbeing scale for farmers in Northeast Thailand consists of 12 factors and 49 indicators. Since the content validity, structure validity and reliability were thoroughly tested, developers or planners can use the wellbeing measurement scale with confidence (measurement consistency, $\propto=0.93$ ) to be used as a tool in constructing or developing the wellbeing of farmers in Northeast Thailand.

\section{ACKNOWLEDGEMENTS}

The authors thank all the participants for their contributions and interest in the project and Mr. Bryan Roderick Hamman and Mrs. Janice Loewen-Hamman for assistance with the English-language presentation of the manuscript.

\section{AUTHOR INFORMATION}

Banjong Krobbuaban, PhD in Development Science by Faculty of Humanity and Social Science Khon Khan, University, Khon Khan, Thailand. Degree in Medical Doctor by Faculty of Medicine Ramathibodi Hospital, Mahidol University, Bangkok, Thailand. Master in Development Strategy by Rajjapat Chaiyaphum University, Thailand. Currently Medical Physician, Expert Level at Chaiyaphum Hospital, Thailand. E-mail: albkb@diamond.mahidol.ac.th. Corresponding author.

Buapun Phompakping, $\mathrm{PhD}$ in Economic and International Development by University of Bath, England. Currently Associate Professor in Faculty of Humanities and Social Sciences, Khon Kaen University, Thailand. Committee of Research and Training Center for Enhancing Quality of Life of Working-Age People, Khon Kaen University, Thailand. E-mail: buapun@kku.ac.th

\section{REFERENCES}

1. Comrey, A.L. and Lee, H. B. (1992). A first course in factor analysis. Hillsdale, New Jersey: Erlbaum.

2. Ganasoot, Prakong. (1995). Statistics for Research in Behavioral Sciences. 2nd ed. Bangkok: Chulalongkorn University Printing House.

3. Gosa, Boonsong. 1999. Physical Education Evaluation and Assessment. Bangkok: Kasetsart University, Bangkhen Campus.

4. Kheawyoo, Jiraporn. (1997). Principle Components and Factor Analysis. Khon Kaen: The Department of Biotatistics and Demography, the Faculty of Public Health, Khon Kaen University.

5. Krobbuaban B, Pompakping B. (2011). Meaning and indicator of Wellbeing in the Aspect of Health of the Farmers in the Northeast of Thailand. J Med Assoc Thai, Vol 94(2), pp.242-249.

6. Layard R. (2005). "Happiness: Lessons from a new science." New York: The Penguin.

7. Luecha, Yuwadee, et al. (1998). Nursing Research Manual. Bangkok: Victory Power Point.

8. Panpruek, Wilawan. (2003). Applied Statistics for Behavioral Sciences. Khon Kaen: The Faculty of Nursing, Khon Kaen University.

9. $\quad$ Pett et al. (2003).Make sense of factor analysis: The use of factor analysis for instrument development in health care research. California: SAGE.

10. Prasitratasin, Suchart. (1997). Research Methodology for Social Sciences. 10th ed. Bangkok: Liang Chiang.

11. Sen, A. (1999). Development as Freedom. Oxford: Oxford University Press. 
NOTES 